\title{
Comparisons of Comorbidities in Patients with Ankylosing Spondylitis and Psoriatic Arthritis and their Influence on Psoriatic Arthritis Disease Activity: Results of a Multicentre Longitudinal Study
}

Carlos Montilla ( montillamorales.carlos@gmail.com )

Hospital Universitario de Salamanca https://orcid.org/0000-0003-2258-2868

MARIA ELISA ACOSTA

Hospital Universitario de Salamanca https://orcid.org/0000-0002-7958-2052

Luis Gómez-Lechón

Hospital Francesc de Borja

Guadalupe Manzano

Hospital Virgen de la Concha

Esther Toledano

Hospital Clinico Universitario San Carlos

Olga Compán

Hospital Universitario de Salamanca

\section{Sonia Pastor}

Hospital Universitario de Salamanca

Cristina Hidalgo

Hospital Universitario de Salamanca

Rubén Queiró

Hospital Universitario Central de Asturias

Research article

Keywords: Psoriatic arthritis, Comorbidity, Obesity, Ankylosing Spondylitis (AS), Depression

Posted Date: January 29th, 2021

DOl: https://doi.org/10.21203/rs.3.rs-156808/v1

License: (1) This work is licensed under a Creative Commons Attribution 4.0 International License.

Read Full License 


\section{Abstract}

Objectives: To compare the presence of psycho-affective disorders and obesity-associated factors between ankylosing spondylitis (AS) and psoriatic arthritis (PsA) patients and to corelate these comorbidities with PsA disease activity.

Methods: This prospective observational longitudinal study included 216 PsA patients and 114 AS patients. Anxiety and depression were compared between both patient groups using the Hospital Anxiety and Depression Scale (HADS) questionnaire. We compared the waist/hip ratio, body mass index (BMI) and laboratory variables including apolipoprotein $A$, apolipoprotein $B$, lipoprotein $A$, the ApoB/ApoA ratio, peptide $C$, insulin, resistance to insulin (HOMA-IR) and leptin. PsA activity was evaluated by the Disease Activity Index for Psoriatic Arthritis (DAPSA) score every 4 months for a year. Patients were divided into two groups: those with moderate or high activity and those with low activity or remission. We compared anxiety-, depression- and obesity-related factors between these two groups.

Results: There were no differences in baseline characteristics between AS and PsA patients. Among the studied factors, only serum leptin was significantly higher in PsA patients than in AS patients (18.49 \pm 19.01 vs $11.5 \pm 10.05, p<0.01)$. Initial serum leptin, obesity and depressive behaviour were poor prognostic factors for persistent low disease activity. The leptin level was correlated with the visual analogue scale (VAS) pain score $(R=0.21, p<0.01)$, VAS activity score $(R=0.26, p<0.01)$, swollen joint count (SJC) $(R=0.23, p<0.01)$ and tender joint count $(T J C)(R=0.26, p<0.01)$.

Conclusion: Obesity alone or concurrent with increased leptin secretion and depression may influence persistent PsA activity. Leptin levels differentiated PsA patients from AS patients.

\section{Key Messages}

- PsA patients are not more obese than AS patients, however high serum leptin levels were more frequent in PsA.

- Obesity and leptin levels were associated with higher PsA disease activity, as leptin influenced the disease activity, affecting pain and inflammation associated variables.

- Depression was also associated with higher disease PsA activity.

\section{Introduction}

Spondyloarthropathies form a group of diseases that share similar immunogenetic, clinical and radiological characteristics [1]. Spondyloarthropathies are differentiated according to these characteristics. For example, psoriatic arthritis (PsA) has a weaker association with HLA-B27 and a lower frequency of axial manifestations than other related diseases; moreover, PsA has specific radiological features. 
Studies comparing the frequencies of comorbidities in patients with different spondyloarthropathies are scarce; therefore, we there is little knowledge about whether comorbidities associated with diseases in this group serve as differentiating or unifying factors. The most frequent comorbidities in PsA patients are emotional disorders and obesity [2]. PsA patients have a higher rate of anxiety and/or depression than psoriasis patients [3-8]. In addition to their associations with limited functionality and a decrease in quality of life, anxiety and depression have been associated with inflammatory activity in patients with these diseases [5]. Up to one-third of patients with ankylosing spondylitis (AS) have depression and/or anxiety, which have been related to increased disease activity $[9,10]$. Obesity is more frequent in PsA patients than in the general population [11]. In addition to being a cardiovascular risk factor, it predisposes psoriasis patients to arthritis development and is associated with a poor response to biologic disease modifying anti-rheumatic drugs (bDMARDs) $[5,6,12]$. Obesity seems to be less frequent in AS patients than in PsA patients; however, it can affect treatment response $[13,14]$. The increase in visceral fat in obese people increases adipokine secretion; adipokines are cytokines that not only influence lipid metabolism and insulin resistance but also possess pro-inflammatory properties [15]. One of these adipokines, leptin, promotes the synthesis of type I pro-inflammatory cytokines and inhibits the synthesis of type II pro-inflammatory cytokines $[16,17]$. Because of these properties, it has been related to inflammatory joint diseases such as rheumatoid arthritis and spondyloarthropathies [18]. Currently, there are no studies that directly compare leptin levels among these pathologies. In patients with AS, there does not appear to be a relationship between serum leptin level and disease activity; however, serum leptin has been associated with radiographic progression $[19,20]$. The concentration of serum leptin is higher in PsA patients than in psoriasis patients, but there are no data to confirm a relationship with disease activity [21].

In the present study, we compared the presence of psycho-affective disorders and factors associated with obesity in patients with AS and PsA. We correlated these factors with persistent clinical activity in PsA patients.

\section{Methods}

A prospective longitudinal observational study was carried out in the rheumatology outpatient clinics of Salamanca, Zamora and Gandía (Valencia) hospitals from November 2018 to December 2019.

Study population

- The inclusion criteria were as follows: consecutive patients who agreed to participate in the study and who were diagnosed with AS according to the New York criteria or PsA according to the Classification Criteria for Psoriatic Arthritis (CASPAR) [22, 23]. The study was carried out with the consent of the ethics committee of the Hospital Clínico Universitario de Salamanca (Ref 19/2018).

- The exclusion criteria were as follows: patients with diabetes mellitus, dyslipidaemia or thyroid disease who were receiving treatment. These patients were excluded due to possible interference with laboratory tests. 
Patients with PsA were followed for a year. PsA activity was assessed by calculating the clinical Disease Activity Index for Psoriatic Arthritis (cDAPSA) score every four months. Two groups were established: those who had a DAPSA score indicating moderate or high activity and those with a score indicating remission or low activity in the three determinations [24]. We compared PsA patients with AS patients and both groups of PsA patients.

Measurement variables

- The following baseline characteristic data were collected: age, sex, disease duration, presence of dactylitis, onychopathy and treatment received. Clinical disease forms (peripheral, axial or mixed) were determined in PsA patients. We considered axial disease in patients with inflammatory low back pain and radiographic sacroiliitis and/or syndesmophytes [25].

- At the beginning of the study, anxiety and depression was measured using the Hospital Anxiety and Depression Scale (HADS) questionnaire [26], which comprises a 14-item scale designed to identify anxiety and depression among individuals with medical conditions. Scores for each subscale (HADSD for depression and HADS-A for anxiety) range from 0-21 and can be classified into 3 categories: normal (0-7), borderline abnormal indicating a possible clinical disorder (8-10), and abnormal indicating a probable clinical disorder.

- Regarding obesity-associated factors, we measured body mass index (BMI), classifying patients as normal, overweight, and obese; the waist/hip ratio; and laboratory variables including apolipoprotein $A$, apolipoprotein $B$, lipoprotein $A$, the $A p o B / A p o A$ ratio, peptide $C$, insulin, insulin resistance (using the homeostasis model assessment for insulin resistance (HOMA-IR) score) and leptin. Leptin and insulin were measured by ELISA (International Standard of the WHO/NIBSC 97/594). The system information was as follows: Cobas e411 analyser with test number 650; Modular Analytics E170 analyser, Cobas e601 and Cobas e602 analysers with application code number 120. The HOMA-IR was calculated as follows: fasting insulin $(\mathrm{mU} / \mathrm{l}) \times$ fasting glucose $(\mathrm{mmol} / \mathrm{I}) / 22.5$. Due to the determination circadian variation, all samples were collected on the same schedule.

\section{Statistical analysis}

Continuous variables are presented as means (Ms) with standard deviations (SDs), and categorical variables are presented as numbers $(\mathrm{N})$ and proportions (\%). Comparisons between two groups were analysed using the $\chi^{2}$ test for categorical variables, $t$ test for continuous variables with normal distributions and the Mann-Whitney $U$ test for continuous variables with nonnormal distributions. For the group with persistently moderate/high DAPSA scores, multivariate analysis was performed by binary logistic regression considering variables with significant differences in the univariate analysis. For continuous variables (leptin, swollen joint count (SJC), tender joint count (TJC) and visual analogue scale (VAS) pain score), bivariate correlations were made using Pearson's correlation coefficients ( $P$ values were considered significant at $<0.05)$. The variables that were significant were analysed using separate stepped simple linear regression models adjusted for sex and treatment, with the SJC, TJC and VAS pain 
score as dependent variables and serum leptin level as the covariable. All statistical analyses were performed using SPSS version 20.

\section{Results}

\section{General characteristics}

A total of 216 patients diagnosed with PsA and 114 patients diagnosed with AS were included.

The PsA patients had a mean age of 52.57 years (SD: 9.95), with a mean disease duration of 10.91 years (SD: 7.9). One hundred thirty-three patients (61.6\%) were men; 10 (4.6\%) had exclusive axial involvement, $73(33.8 \%)$ had mixed axial involvement, and the rest had only peripheral involvement. Thirty-eight patients (17.6\%) had dactylitis, and 101 had onychopathy (46.8\%). Regarding treatment with synthetic disease modifying anti-rheumatic drugs (DMARDs), 85 patients (39.4\%) received methotrexate treatment, 39 (18.1\%) received salazopyrin, 11 (5.1\%) received leflunomide, and the rest had not received DMARDs. Thirty-four patients (15.7\%) received biological DMARDS (bDMARDs), and 3 (1.4\%) received apremilast. Regarding comorbidities, 85 patients (39.4\%) had a normal BMI, 83 (38.4\%) were overweight, and 48 (22.2\%) were obese. According to the HADS-A questionnaire, 37 (17.1\%) patients were classified as having possible anxiety, and $43(19 \%)$ were classified as having probable anxiety. Furthermore, 33 (15.3\%) patients had possible depression, and 18 (8.3\%) had probable depression.

The mean age of AS patients was 50.40 years (SD: 10.64), with a mean disease duration of 14.21 years (SD: 8.98). Sixty-eight patients were men (59.6\%). Ninety-two (80.7\%) patients had exclusive axial involvement, and 17 (14.9\%) had dactylitis. Regarding treatment, $13(11.4 \%)$ patients received salazopyrin treatment, and 9 (7.9\%) received methotrexate. Twenty-five $(21.9 \%)$ patients had received DMARD treatment. Regarding comorbidities, 47 (41.2\%) had a normal BMI, 50 (43.9\%) were overweight, and 17 (14.9\%) were obese. According to the HADS-A questionnaire, 17 (14.9\%) patients were classified as having possible anxiety, and $26(22.8 \%)$ were classified as having probable anxiety. According to the HADS-D questionnaire, 15 (13.2\%) patients were classified as having possible depression, and 11 (9.6\%) were classified as having probable depression.

\section{Comparisons between the PsA and AS groups}

We did not find any differences in the baseline characteristics between the two disease groups (Table 1). Regarding laboratory parameters associated with obesity, we found a higher serum leptin concentration in the PsA group than in the AS group (18.48 \pm 19.01 vs $11.84 \pm 10.23, p<0.01)$. When adjusted for sex ( $p$ $<0.001$; OR: $1.04 ; 95 \% \mathrm{Cl}: 1.02-1.07)$. We found no differences in the HADS-A (6.90 \pm 4.60 vs $6.84 \pm 4.25$; $p=0.9)$ and HADS-D $(4.69 \pm 4.05$ vs $4.62 \pm 3.85)$ questionnaire results between groups. Other differences are shown in Table 1. 
Table 1

Comorbidity comparisons between AS and PsA patients

\begin{tabular}{|c|c|c|c|c|}
\hline & AS & PsA & $\mathbf{p}$ & $\begin{array}{l}\text { Multivariate } \\
\text { analysis }\end{array}$ \\
\hline Age: mean (SD) & $50.40(10.64)$ & $52.57(9.95)$ & 0.4 & \\
\hline Disease duration: mean (SD) & $14.21(8.98)$ & $10.91(7.99)$ & 0.3 & \\
\hline Sex: Male/Female & $68 / 46$ & $133 / 83$ & 0.7 & \\
\hline bDMARD: (\%) & 21.9 & 15.7 & 0.7 & \\
\hline $\begin{array}{l}\text { Abdominal circumference: mean } \\
\text { (SD) }\end{array}$ & $88.99(12.73)$ & $90.49(13.78)$ & 0.3 & \\
\hline Hip circumference: mean (SD) & $97.36(8.40)$ & $98.20(8.85)$ & 0.4 & \\
\hline Waist/hip ratio: mean (SD) & $0.91(0.09)$ & $0.92(0.1)$ & 0.5 & \\
\hline BMI: mean (SD) & $26.09(3.10)$ & $26.79(3.51)$ & 0.09 & \\
\hline Normal/Overweight/Obese: (\%)* & 41.2/43.9/14.9 & $39.4 / 38.4 / 22.2$ & 0.2 & \\
\hline \multirow[t]{2}{*}{ Leptin (ng/ml): mean (SD) } & $11.5(10.05)$ & $18.49(19.01)$ & 0.008 & $\begin{array}{l}\mathrm{p}<0.001 \\
\text { OR:1.04 }\end{array}$ \\
\hline & & & & $\begin{array}{l}\text { (95\% Cl: } 1.02- \\
1.07)^{\star}\end{array}$ \\
\hline Peptide C (ng/ml): mean (SD) & $2.40(0.95)$ & $2.66(1.21)$ & 0.1 & \\
\hline Insulin $(\mu \mathrm{U} / \mathrm{mL})$ : mean $(\mathrm{SD})$ & $9.88(5.55)$ & $11.22(7.98)$ & 0.3 & \\
\hline HOMA-IR: mean (SD) & $2.83(4.71)$ & $2.83(2.91)$ & 0.1 & \\
\hline ApoA (mg/dL): mean (SD) & $159.28(24.51)$ & $161.25(26.89)$ & 0.6 & \\
\hline ApoB (mg/dL): mean (SD) & $100.80(24.40)$ & $97.66(25.12)$ & 0.4 & \\
\hline Lipoprotein A (nmol/L): mean (SD) & $49.61(63.66)$ & $50.11(59.43)$ & 0.8 & \\
\hline ApoB/ApoA: mean (SD) & $0.69(0.25)$ & $0.68(0.31)$ & 0.1 & \\
\hline HADS-A: mean (SD) & $6.90(4.60)$ & $6.84(4.25)$ & 0.9 & \\
\hline $\begin{array}{l}\text { HADS-A: } \\
\text { normal/possible/probable: \% }\end{array}$ & $62.3 / 14.9 / 22.8$ & 63/17.1/19.9 & 0.7 & \\
\hline HADS-A: mean (SD) & $4.69(4.05)$ & $4.62(3.80)$ & 0.9 & \\
\hline $\begin{array}{l}\text { HADS-D: } \\
\text { normal/possible/probable: } \%\end{array}$ & 77.2/13.2/9.6 & 76.4/15.3/8.3 & 0.8 & \\
\hline *Adjusted for sex & & & & \\
\hline
\end{tabular}




\section{Disease activity associations with obesity and its related factors and HADS questionnaire results}

Thirty-eight patients $(17.6 \%)$ had moderate activity at all three time points. Patients with persistent moderate disease activity according to the DAPSA score had a higher rate of obesity $(28.2 \%$ vs $8.8 \%$; $p<$ $0.006)$, had a higher leptin serum concentration $(31.60 \pm 24.71$ vs $24.34 \pm 23.19 ; p<0.001)$ and had higher HADS-A ( $8.75 \pm 4.04$ vs $6.40 \pm 4.19 ; p<0.001)$ and HADS-D scores $(7.07 \pm 4.47$ vs $4.13 \pm 3.50 p<0.001)$ than patients with low or no disease activity. In the multivariate analysis adjusted for sex and biological treatment, leptin ( $p<0.01$; OR: 1.03; 95\% Cl: 1.013-1.057), depression ( $<<0.03$; OR: 1.18; 95\% Cl: 1.013$1.395)$ and obesity ( $<<0.01$; OR: $2.87: 95 \% \mathrm{Cl}: 1.04-7.92)$ influenced disease activity. The Nagelkerke $\mathrm{R}^{2}$ was 0.26 . The other results are shown in Table 2. 
Table 2

Baseline variables and comorbidity relationships with DAPSA score

\begin{tabular}{|c|c|c|c|c|}
\hline & DAPSA $\leq 14$ & DAPSA $\geq 15$ & $\mathbf{p}$ & $\begin{array}{l}\text { Multivariate } \\
\text { analysis }\end{array}$ \\
\hline Age: mean (SD) & $51.98(9.89)$ & $55.15(9.91)$ & 0.2 & \\
\hline Disease duration: mean (SD) & $10.76(8.18)$ & $11.57(7.13)$ & 0.2 & \\
\hline Sex: Male/Female & $113 / 63$ & $20 / 20$ & 0.09 & \\
\hline bDMARD: \% & 15.3 & 17.5 & 0.7 & \\
\hline BMI: mean (SD) & $26.54(3.19)$ & $26.74(3.29)$ & 0.6 & \\
\hline \multirow[t]{2}{*}{ Normal/Overweight/Obese: \%* } & $30.4 / 60.8 / 8.8$ & $33.3 / 38.5 / 28.2$ & 0.006 & $\begin{array}{l}\mathrm{p}<0.04 ; \text { OR: } \\
\text { 2.87: }\end{array}$ \\
\hline & & & & $\begin{array}{l}95 \% \mathrm{Cl}: 1.04- \\
7.92\end{array}$ \\
\hline Waist/hip ratio: mean (SD) & $0.92(0.11)$ & $0.92(0.12)$ & 0.9 & \\
\hline \multirow[t]{2}{*}{ Leptin (ng/ml): mean (SD) } & $15.50(16.11)$ & $31.60(24.71)$ & 0.001 & $\mathrm{p}<0.01$; OR: 1.03; \\
\hline & & & & $\begin{array}{l}95 \% \mathrm{Cl}: 1.013- \\
1.057\end{array}$ \\
\hline Peptide C (ng/ml): mean (SD) & $2.52(1.13)$ & $2.82(1.16)$ & 0.06 & NS \\
\hline Insulin $(\mu \mathrm{U} / \mathrm{mL})$ : mean $(\mathrm{SD})$ & $10.65(7.71)$ & $12.33(7.98)$ & 0.05 & NS \\
\hline HOMA-IR: mean (SD) & $2.59(2.14)$ & $2.85(1.91)$ & 0.07 & NS \\
\hline ApoA (mg/dL): mean (SD) & $\begin{array}{l}162.04 \\
(27.24)\end{array}$ & $158.74(24.86)$ & 0.6 & \\
\hline ApoB (mg/dL): mean (SD) & $96.34(23.07)$ & 100.09 (30.02) & 0.2 & \\
\hline Lipoprotein A (nmol/L): mean (SD) & $51.69(61.73)$ & $45.53(50.95)$ & 0.5 & \\
\hline ApoB/ApoA: mean (SD) & $0.61(0.18)$ & $0.64(0.22)$ & 0.3 & \\
\hline HADS-A: mean (SD) & $6.40(4.19)$ & $8.75(4.04)$ & 0.001 & NS \\
\hline $\begin{array}{l}\text { HADS-A: } \\
\text { normal/possible/probable: \% }\end{array}$ & $67 / 17 / 16$ & $45 / 17.5 / 37.5$ & 0.006 & \\
\hline
\end{tabular}

* Patients were categorized into two groups: those with DAPSA scores $<14$ (remission or low activity) and those with DAPSA scores $>15$ (moderate or high activity)

** The multivariate analysis was adjusted for sex and biological treatment with the presence or absence of obesity, leptin level and the results of the HADS questionnaire for both anxiety and depression. 


\begin{tabular}{|c|c|c|c|c|}
\hline & DAPSA $\leq 14$ & DAPSA $\geq 15$ & $\mathbf{p}$ & $\begin{array}{l}\text { Multivariate } \\
\text { analysis }\end{array}$ \\
\hline \multirow[t]{2}{*}{ HADS-A: mean (SD) } & $4.13(3.50)$ & $7.07(4.47)$ & 0.001 & $p<0.03 ;$ OR:1.18; \\
\hline & & & & $\begin{array}{l}\text { IC 95\%: } 1.013- \\
1.395\end{array}$ \\
\hline $\begin{array}{l}\text { HADS-D: } \\
\text { normal/possible/probable: \% }\end{array}$ & $81.8 / 11.4 / 6.8$ & $52.5 / 35.5 / 15$ & 0.001 & \\
\hline \multicolumn{5}{|c|}{$\begin{array}{l}\text { * Patients were categorized into two groups: those with DAPSA scores }<14 \text { (remission or low activity) } \\
\text { and those with DAPSA scores }>15 \text { (moderate or high activity) }\end{array}$} \\
\hline \multicolumn{5}{|c|}{$\begin{array}{l}\text { ** The multivariate analysis was adjusted for sex and biological treatment with the presence or } \\
\text { absence of obesity, leptin level and the results of the HADS questionnaire for both anxiety and } \\
\text { depression. }\end{array}$} \\
\hline
\end{tabular}

Leptin levels were correlated with the VAS pain score (R: $0.21, p<0.01)$, VAS activity score (R: $0.26, p<$ $0.01)$, SJC (R: 0.23, p<0.01), and TJC (R: 0.26, p<0.01).

In the linear regression analysis, which was performed separately and adjusted for sex and treatment, the SJC, TJC, VAS pain score and VAS activity score were the dependent variables, and leptin serum concentration was the independent variable. We obtained the following results: SJC: $\boldsymbol{\beta}: 0.13 ; p<0,001$; 95\% Cl: 0.006-0.21; $\mathrm{R}^{2}$ : 0.05; TJC: $\boldsymbol{\beta}: 0.15 ; p<0.001 ; 95 \%$ Cl: $0.008-0.22 ; R^{2}$ : 0.07; pain VAS score: $\boldsymbol{\beta}: 0.03$; $p<0.002 ; 95 \%$ Cl: $\left.0.01-0.05 ; R^{2}: 0.04\right)$ and VAS activity score: $\boldsymbol{\beta}: 0.04 ; p<0,001 ; 95 \%$ Cl: $0.021-0.05 ; R^{2}$ : $0.07)$.

There were also relationships between leptin level and abdominal circumference (R: 0.24, $p<0.001$ ), hip circumference (R: 0.56, $p<0.001)$ and BMI (R: 0.14, $p<0.03)$.

Leptin levels in patients with remission according to the DAPSA score were $13.10 \pm 10.86(\mathrm{ng} / \mathrm{ml})$, in patients with low activity were $16.34 \pm 18.44(\mathrm{ng} / \mathrm{ml})$ and in patients with moderate activity were $27.85 \pm$ $23.18(\mathrm{ng} / \mathrm{ml})$. There were significant differences in leptin levels between patients with moderate activity and patients with remission $(p<0.001)$ and between patients with moderate activity and low activity $(p<$ $0.001)$.

\section{Discussion}

In our study, metabolic factors had a greater influence on disease activity than emotional disorders in PsA patients, and this influence was greater in PsA patients than in AS patients. The differences were associated with laboratory factors associated with obesity (leptin concentration), not obesity itself. Obesity, leptin levels, and depression were associated with increased disease activity. Leptin influenced disease activity, affecting pain- and inflammation-associated variables. 
We found no differences in the presence of anxiety and/or depression between the patients with PsA and the patients with AS. Only one other study directly compared patients with PsA and other spondyloarthropathies using the HADS questionnaire. The authors also found no differences, and the mean HADS questionnaire scores were similar to those in our study [10].

The prevalence of obesity is higher in PsA patients than in the general population [11]. Although PSA patients have a higher rate of obesity than AS patients, few studies have compared this comorbidity between these patient groups. Haque et al. [27] demonstrated that PsA patients had a higher metabolic rate than patients with other spondyloarthropathies. This difference was determined based on a greater abdominal circumference (102.46 vs $98.29 p<0.02)$ and higher triglyceride level $(163.07$ vs $133.47 ; p<$ 0.001) in patients with other spondyloarthropathies [27]. We did not find differences in the waist/hip ratio, abdominal circumference, or categorized BMI between the PsA and AS groups. The difference between our results and those published previously could be due to genetic or sociocultural factors since in Haque's study, both PsA and AS patients had greater abdominal circumferences than the patients in our study (102.46 vs 90.49 and 98.29 vs 89.89 , respectively). The exclusion of patients with diabetes or dyslipidaemia receiving treatment in our study could also explain this difference.

Leptin is a hormone secreted by adipocytes that acts on various metabolic pathways regulating body weight; it also has pro-inflammatory properties, as it intervenes in cellular immunity, activates the Th1 response and increases IL-1 $\beta$, IL- 6 and TNF-a levels $[15,16]$. There are no other studies comparing leptin levels between patients with PsA and AS. In previous studies involving AS patients, serum leptin levels fluctuated substantially and were similar to those found in the control group $(7.2-17.2)[18,27]$. In a study carried out in a Spanish population, the results were very similar to those in our study (8.5 vs 8.9 in men and 18.9 vs 17.01 in women) [28]. In our study, the leptin level, obesity, and depression predicted persistent clinical PsA activity. Recently, Lubrano et al. [29] demonstrated that the number of comorbidities, including general comorbidities, anxiety and fibromyalgia in particular, had an impact on the DAPSA scores. Other comorbidities, such as depression, diabetes and obesity, did not influence activity [29].

Several studies have specifically linked some comorbidities with disease activity. McDonough et al. [5] found a relationship between anxiety/depression measured by the HADS questionnaire and the SJC. This relationship can be explained in two ways: functionality limitation and loss secondary to inflammation could cause depression or anxiety, or psychologic stress affecting the hypothalamic-pituitary-adrenal axis could stimulate T-cells, promoting the secretion of cytokines (TNF-a, IL-1) [30]. For this reason, biologic treatment may improve anxiety and depressive symptoms regardless of the particular effect [31]. In our study, the rates of probable anxiety and depression were similar to those previously published. We also found a significant correlation between the SJC and HADS scores for both anxiety and depression (data not shown) [5].

Several reports support a link between obesity and disease activity. Thus, weight loss has been associated with an improved response to anti-TNF-a therapy. Moreover, obese patients are less likely to 
achieve minimal disease activity (MDA) than non-obese patients [32,33]. Reports have shown that obesity influences only the pain parameter of the MDA score [33].

There are several studies on the influence of leptin on PsA disease activity. Eder et al. [21] did not find a significant correlation between leptin level and the SJC, although they did find a strong trend (p: 0.05). A study in Asian patients found an association between the leptin level and the Psoriatic Arthritis Joint Activity Index (PsAJAI); however, the authors did not analyse relationships with the different components of the index. Therefore, we do not know if the leptin level was correlated with inflammation-associated parameters [34]. A recent study revealed associations between leptin level and the VAS score and TJC [35], and other results also support the theory that leptin may be a pain modulator [36, 37]. In our study, leptin was correlated with both the SJC and TJC, suggesting it influenced inflammatory and pain variables.

As previously described, we also found a relation between BMI and leptin levels [21, 35]. In some studies on rheumatoid arthritis, leptin was correlated with disease activity, although it was not associated with BMI $[38,39]$. This dissociation may be related to the fact that leptin synthesis does not occur in only abdominal fat. In osteoarthritis patients, increased leptin secretion has been found in adipocytes in infrapatellar fat [40].

One of our study limitations was that we did not compare leptin concentrations between disease groups and a control group; however, previous studies have found higher serum levels in PsA patients than in healthy subjects [34].

\section{Conclusions}

In conclusion, PsA patients do not have a higher rate of obesity than AS patients; however, some metabolic factors, such as a high serum leptin level, were more frequent in these patients. Obesity and depression as comorbidities and increased leptin possibly secondary to obesity can serve as predictors of persistent moderate/high disease activity. These results are relevant in clinical practice, as comorbidities (obesity, emotional factors) should be taken into account when managing disease activity and comorbidities, as they could affect therapeutic decisions.

\section{Declarations}

- Ethical Approval and Consent to participate: The study was carried out with the consent of the ethics committee of the Hospital Clínico Universitario de Salamanca (Ref 19/2018).

- Consent for publication: Not applicable

- Availability of supporting data: The datasets used and/or analyzed during the current study are available from the corresponding author on reasonable request.

- Competing interests: The authors declare that they have no competing interests

- Funding: None 
- Authors' contributions: CM substantially contributed to the acquisition of data, performed the statistical analysis, critically interpreted the results, and wrote the first draft of the manuscript. LGL contributed to the acquisition of data, critically interpreted the results, and revised the manuscript. GM contributed to the acquisition of data, critically interpreted the results, and revised the manuscript. MEA, ET, OC, SP, $\mathrm{CH}$, and RQ critically interpreted the results and revised the manuscript. All authors read and approved the final version of the manuscript.

- Acknowledgements: None

- Authors' information: CM, MD, PhD. MEA, MD. LGL, MD. GM, MD. ET, MD, PhD. OC, MD. SP, MD. CH, MD. RQ, MD, PhD.

\section{References}

1. Van der Linden S, Van Der Heijden D. Classification of spondyloarthropathies. In: Hochberg M, Gravallese E, Silman A, Smolen J, Weinblatt M, Weisman M, editors. Rheumatology. Edinburgh: Mosby; 2003. pp. 1149-51.

2. Raychaudhuri SP. Comorbidities of psoriatic arthritis - metabolic syndrome and prevention: a report from the GRAPPA 2010 annual meeting. $J$ Rheumatol 2012;39:437 - 40.

3. Kotsis K, Voulgari PV, Tsifetaki N, et al. Anxiety and depressive symptoms and illness perceptions in psoriatic arthritis and associations with physical health-related quality of life. Arthritis Care Res. 2012;64:1593-601.

4. Husted JA, Thavaneswaran A, Chandran V, Gladman DD. Incremental effects of comorbidity on quality of life in patients with psoriatic arthritis. J Rheumatol. 2013;40:1349-56.

5. McDonough E, Ayearst R, Eder L, et al. Depression and anxiety in psoriatic disease: prevalence and associated factors. J Rheumatol. 2014;41:887-96.

6. Micheroli R, Hebeisen M, Wildi LM, et al. Impact of obesity on the response to tumor necrosis factor inhibitors in axial spondyloarthritis. Arthritis Res Ther. 2017;19:164.

7. Harvima RJ, Viinamäki H, Harvima IT, et al. Association of psychic stress with clinical severity and symptoms of psoriatic patients. Acta Derm Venereol. 1996;76:467-71.

8. Freire M, Rodríguez J, Möller I, et al. Prevalence of symptoms of anxiety and depression in patients with psoriatic arthritis attending rheumatology clinics. Reumatol Clín (Engl Ed). 2011;7:20-6.

9. Kilic G, Kilic E, Ozgocmen S. Relationship between psychiatric status, self-reported outcome measures, and clinical parameters in axial spondyloarthritis. Medicine. 2014;93:e337.

10. Chan CYY, Tsang HHL, Lau CS, Chung HY. Prevalence of depressive and anxiety disorders and validation of the Hospital Anxiety and Depression Scale as a screening tool in axial spondyloarthritis patients. Int J Rheum Dis. 2017;20:317-25.

11. Peters MJ, van der Horst-Bruinsma IE, Dijkmans BA, Nurmohamed MT. Cardiovascular risk profile of patients with spondylarthropathies, particularly ankylosing spondylitis and psoriatic arthritis. Semin Arthritis Rheum. 2004;34:585-92. 
12. Soltani-Arabshahi R, Wong B, Feng BJ, Goldgar DE, Duffin KC, Krueger GG. Obesity in early adulthood as a risk factor for psoriatic arthritis. Arch Dermatol. 2010;146:721-6.

13. Masi AT, Aldag JC, Mohan PC, Murugan TSR. Significantly lower serum triglyceride levels in ankylosing spondylitis patients than age-, gender-, and medical service-matched controls: results of multivariate analysis. Arthritis Rheum. 2000;43:104.

14. Singh S, Facciorusso A, Singh AG, et al. Obesity and response to anti-tumor necrosis factor-a agents in patients with select immune-mediated inflammatory diseases: a systematic review and metaanalysis. PLoS One. 2018;13:e0195123.

15. Russolillo A, lervolino S, Peluso R, et al. Obesity and psoriatic arthritis: from pathogenesis to clinical outcome and management. Rheumatology. 2013;52:62-7.

16. Johnston A, Arnadottir S, Gudjonsson JE, et al. Obesity in psoriasis: leptin and resistin as mediators of cutaneous inflammation. Br J Dermatol. 2008;159:342-50.

17. Abella V, Scotece M, Conde J, et al. Leptin in the interplay of inflammation, metabolism and immune system disorders. Nat Rev Rheumatol. 2017;13:100-9.

18. Abella V, Scotece M, Conde J, et al. Adipokines, metabolic syndrome and rheumatic diseases. J Immunol Res. 2014;2014:343746.

19. Sari I, Demir T, Kozaci LD, et al. Body composition, insulin, and leptin levels in patients with ankylosing spondylitis. Clin Rheumatol. 2007;26:1427-32.

20. Hartl A, Sieper J, Syrbe U, et al. Serum levels of leptin and high molecular weight adiponectin are inversely associated with radiographic spinal progression in patients with ankylosing spondylitis: results from the ENRADAS trial. Arthritis Res Ther. 2017;19:140.

21. Eder L, Jayakar J, Pollock R, et al. Serum adipokines in patients with psoriatic arthritis and psoriasis alone and their correlation with disease activity. Ann Rheum Dis. 2013;72:1956-61.

22. Van Der Linden S, Valkenburg HA, Cats A. Evaluation of diagnostic criteria for ankylosing spondylitis. Arthritis Rheum. 1984;27:361-8.

23. Taylor W, Gladman D, Helliwell P, Marchesoni A, Mease P, Mielants H. Classification criteria for psoriatic arthritis: development of new criteria from a large international study. Arthritis Rheum. 2006;54:2665-73.

24. Schoels MM, Aletaha D, Alasti F, Smolen JS. Disease activity in psoriatic arthritis (PsA): defining remission and treatment success using the DAPSA score. Ann Rheum Dis. 2015;75:811-8.

25. Sieper J, van der Heijde D, Landewe R, et al. New criteria for inflammatory back pain in patients with chronic back pain: a real patient exercise by experts from the Assessment of SpondyloArthritis international Society (ASAS). Ann Rheum Dis. 2009;68:784-8.

26. Zigmond AS, Snaith RP. The hospital anxiety and depression scale. Acta Psychiatr Scand. 1983;67:361-70.

27. Haque N, Lories RJ, de Vlam K. Comorbidities associated with psoriatic arthritis compared with nonpsoriatic spondyloarthritis: a cross-sectional study. J Rheumatol. 2016;43:376-82. 
28. Gonzalez-Lopez L, Fajardo-Robledo NS, Miriam Saldaña-Cruz A, et al. Association of adipokines, interleukin-6, and tumor necrosis factor-a concentrations with clinical characteristics and presence of spinal syndesmophytes in patients with ankylosing spondylitis: a cross-sectional study. J Int Med Res. 2017;45:1024-35.

29. Lubrano E, Scriffignano S, Azuaga AB, Ramirez J, Cañete JD, Perrotta FM. Impact of comorbidities on disease activity, patient global assessment, and function in psoriatic arthritis: a cross-sectional study. Rheumatol Ther. 2020;7:825-36.

30. Dhabhar FS. Enhancing versus suppressive effects of stress on immune function: implications for immunoprotection and immunopathology. Neuroimmunomodulation. 2009;16:300-17.

31. Tyring S, Gottlieb A, Papp K, et al. Etanercept and clinical outcomes, fatigue, and depression in psoriasis: double-blind placebo-controlled randomised phase III trial. Lancet. 2006;367:29-35.

32. Di Minno MND, Peluso R, lervolino S, et al. Weight loss and achievement of minimal disease activity in patients with psoriatic arthritis starting treatment with tumour necrosis factor a blockers. Ann Rheum Dis. 2014;73:1157-62.

33. Eder L, Thavaneswaran A, Chandran V, Cook RJ, Gladman DD. Obesity is associated with a lower probability of achieving sustained minimal disease activity state among patients with psoriatic arthritis. Ann Rheum Dis. 2015;74:813-7.

34. Xue Y, Jiang L, Cheng Q, et al. Adipokines in psoriatic arthritis patients: the correlations with osteoclast precursors and bone erosions. PLoS One. 2012;7:e46740.

35. Bandinelli F, Prignano F, Bonciani D, et al. Clinical and demographic factors influence on anxiety and depression in early psoriatic arthritis (ePsA). Clin Exp Rheumatol. 2013;31:318-9.

36. Lim G, Wang S, Zhang Y, Tian Y, Mao J. Spinal leptin contributes to the pathogenesis of neuropathic pain in rodents. J Clin Investig. 2009;119:295-304.

37. Tian Y, Wang S, Ma Y, Lim G, Kim H, Mao J. Leptin enhances NMDA-induced spinal excitation in rats: a functional link between adipocytokine and neuropathic pain. Pain. 2011;152:1263-71.

38. Bustos Rivera-Bahena C, Xibillé-Friedmann DX, González-Christen J, Carrillo-Vázquez SM, MontielHernández JL. Peripheral blood Leptin and Resistin levels as clinical activity biomarkers in Mexican Rheumatoid Arthritis patients. Reumatol Clín (Engl Ed). 2016;12:323-6.

39. Dervišević A, Resić $H$, Sokolović Š, et al. Leptin is associated with disease activity but not with anthropometric indices in rheumatoid arthritis patients. Arch Med Sci. 2018;14:1080-6.

40. Conde J, Scotece M, López V, et al. Differential expression of adipokines in infrapatellar fat pad (IPFP) and synovium of osteoarthritis patients and healthy individuals. Ann Rheum Dis. 2014;73:631-3. 